\title{
ANALYSIS OF TILTING TABLE WITH PARALLEL KINEMATICS
}

\author{
István Tüske \\ PhD Student, University of Miskolc, Institute of Machine Tools and Mechatronics, Department of Machine Tools \\ 3515 Miskolc, Miskolc-Egyetemváros, e-mail: tuske.istvan@student.uni-miskolc.hu
}

György Hegedüs

associate professor, University of Miskolc, Institute of Machine Tools and Mechatronics,

Department of Machine Tools

3515 Miskolc, Miskolc-Egyetemváros, e-mail: hegedus.gyorgy@uni-miskolc.hu

\begin{abstract}
The paper deals with the investigation of a workpiece moving unit with parallel kinematics. The subject of the study is the choice of the positions, installation location of actuator of the workpiece moving unit, table, how it affects the operation of the structure. This study is extremely important because the position of the actuators determines the functionality of the structure, i.e., we can predefine the position of the actuators so that the system can perform the desired movements. The possible installation locations of the actuators will be determined using combinatorics. Screw theory will be used to calculate the degree of freedom of the mechanism.
\end{abstract}

Keywords: machine tool, parallel kinematics, screw theory, combinatorics, degree of freedom

\section{Introduction}

One of the subtasks of the workpiece moving unit analysis is to explore the installation locations of the platform tilting actuators. This task is related to the degree of freedom of the structure as the position of the actuators affects the mobility of the mechanism. Variations of possible installation locations will be the starting point for determining the degree of freedom for which screw theory will be used (Bas, 2019). The result of the analysis will show where the coordinate points on the table are, where the actuators are installed the degree of freedom of the mechanism is two, i.e., the platform can rotate about the $\mathrm{x}$ and $\mathrm{y}$ axes. The scientific background of the paper is very broad. The solution to the problem is elaborated in several chapters. The second chapter describes screw theory, which requires knowledge of vectors, three literatures have also been used in this section. In order to perform the task, it is necessary to able to describe the exact structure of the analysed mechanism, the third chapter builds on knowledge of parallel mechanism. Combinatorial tools have been used to determine the possible structure of the mechanism during the elaboration of the fourth chapter using two literatures. Furthermore, the fourth chapter defines the degree of freedom of the structure of the mechanism of the given arrangement, which is done using the screw theory described in the second chapter.

\section{Introduction to Screw Theory}

Second chapter is based on (Marshall, 1998; Szeidl, 2014; Huang et al., 2012). Given a line with the location vectors $\boldsymbol{r}_{\boldsymbol{I}}\left(x_{1}, y_{1}, z_{1}\right)$ for point $\mathrm{P}_{1}$ and $\boldsymbol{r}_{2}\left(x_{2}, y_{2}, z_{2}\right)$ for point $\mathrm{P}_{2}$. The direction vector can be written as the difference between the vectors $\boldsymbol{r}_{\mathbf{2}}$ and $\boldsymbol{r}_{\mathbf{1}}$, Figure 1: $\overrightarrow{\boldsymbol{P}_{\mathbf{1}} \boldsymbol{P}_{\mathbf{2}}}=\boldsymbol{r}_{\mathbf{2}}-\boldsymbol{r}_{\mathbf{1}}=\left(x_{2}-x_{1}\right) \boldsymbol{i}+$ 
$\left(y_{2}-y_{1}\right) \boldsymbol{j}+\left(z_{2}-z_{1}\right) \boldsymbol{k}$ is one of the direction vectors of the line, where $\boldsymbol{i}, \boldsymbol{j}$ and $\boldsymbol{k}$ are the base vectors in the $\mathrm{x}, \mathrm{y}$ and $\mathrm{z}$ directions (Marshall, 1998). Then it can be written: $x_{2}-x_{1}=L, y_{2}-y_{1}=M, z_{2}-$ $z_{1}=N . \overrightarrow{\boldsymbol{P}_{\mathbf{1}} \boldsymbol{P}_{\mathbf{2}}}=L \boldsymbol{i}+M \boldsymbol{j}+N \boldsymbol{k}$ takes shape, where $L, M, N$ are the direction ratios. And the length of the vector: $\overrightarrow{\left|\boldsymbol{P}_{\mathbf{1}} \boldsymbol{P}_{\mathbf{2}}\right|}=\sqrt{L^{2}+M^{2}+N^{2}}$. The direction cosines can be expressed in this form: $l=$ $\frac{L}{\mid \overrightarrow{\left|\boldsymbol{P}_{\mathbf{1}} \boldsymbol{P}_{\mathbf{2}}\right|}}, m=\frac{M}{\mid \overrightarrow{\boldsymbol{P}_{\mathbf{1}} \boldsymbol{P}_{2} \mid}}, n=\frac{N}{\left|\boldsymbol{P}_{\mathbf{1}} \boldsymbol{P}_{\mathbf{2}}\right|}$, where $l^{2}+m^{2}+n^{2}=1$. It can be written using any point on the line: $\left(\boldsymbol{r}-\boldsymbol{r}_{1}\right) \times \overrightarrow{\boldsymbol{P}_{\mathbf{1}} \boldsymbol{P}_{\mathbf{2}}}=\mathbf{0}$. For simplicity, $\overrightarrow{\boldsymbol{P}_{\mathbf{1}} \boldsymbol{P}_{\mathbf{2}}}$ denoted by $\boldsymbol{S}$. Then $\left(\boldsymbol{r}-\boldsymbol{r}_{1}\right) \times \boldsymbol{S}=\mathbf{0}, \boldsymbol{r} \times \boldsymbol{S}-\boldsymbol{r}_{\mathbf{1}} \times \boldsymbol{S}=$ 0, i.e., the torque of the line to the origin: $r \times S=r_{1} \times S=S_{0}$.

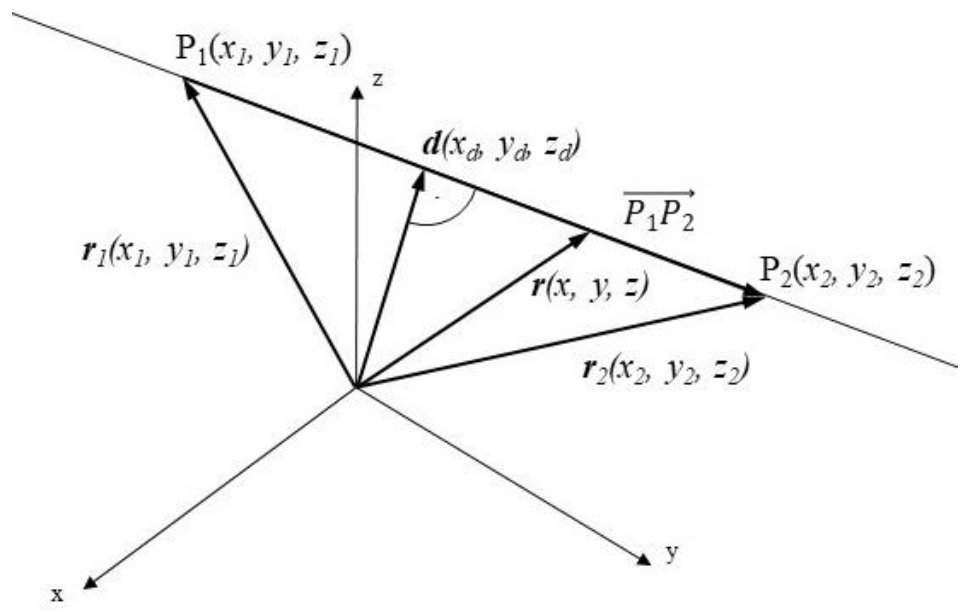

Figure 1. The direction vector of the line.

The vector pair $\left(\boldsymbol{S}, \boldsymbol{S}_{\mathbf{0}}\right)$ is called the Plücker vector of the line and satisfies the condition of orthogonality, $\boldsymbol{S} \cdot \boldsymbol{S}_{\mathbf{0}}=0$. Explaining the $\boldsymbol{r}_{1} \times \boldsymbol{S}=\boldsymbol{S}_{\mathbf{0}}$ relation, it follows that: $\boldsymbol{S}_{0}=\left|\begin{array}{ccc}\boldsymbol{i} & \boldsymbol{j} & \boldsymbol{k} \\ x_{1} & y_{1} & z_{1} \\ L & M & N\end{array}\right|$ and $\boldsymbol{S}_{0}$ can be written in the following form: $\boldsymbol{S}_{0}=P \boldsymbol{i}+Q \boldsymbol{j}+R \boldsymbol{k}$, where $P=y_{1} N-z_{1} M, Q=x_{1} N-z_{1} L$, and $R=x_{1} M-y_{1} L$. The condition of orthogonality takes the following form: $\boldsymbol{S} \cdot \boldsymbol{S}_{\mathbf{0}}=L P+M Q+N R=$ 0 , the Plücker coordinates of the line: $(L, M, N ; P, Q, R)$. The distance of the line from origin is represented by the vector $\boldsymbol{d}$ (Figure 1). Multiply both sides of the equation $\boldsymbol{d} \times \boldsymbol{S}=\boldsymbol{S}_{\mathbf{0}}$ vectorially from left: $\boldsymbol{S} \times(\boldsymbol{d} \times \boldsymbol{S})=\boldsymbol{S} \times \boldsymbol{S}_{\mathbf{0}}$. Arranging the left side of the equation and taking expansion of vector triple product (Szeidl, 2014): $\boldsymbol{S} \times(\boldsymbol{d} \times \boldsymbol{S})=(\boldsymbol{S} \cdot \boldsymbol{S}) \boldsymbol{d}-(\boldsymbol{S} \cdot \boldsymbol{d}) \boldsymbol{S}=(\boldsymbol{S} \cdot \boldsymbol{S}) \boldsymbol{d}$, taking the absolute value of the equation $\boldsymbol{d}=\frac{\boldsymbol{S} \times \boldsymbol{S}_{\mathbf{0}}}{\boldsymbol{S} \cdot \boldsymbol{S}}$, and after simplifications: $|\boldsymbol{d}|=\frac{\left|\boldsymbol{S}_{\mathbf{0}}\right|}{|\boldsymbol{S}|}$. If $\boldsymbol{S}_{\mathbf{0}}=\mathbf{0}$, the line passes through the origin and the Plücker vectors of the line: $(\boldsymbol{S} ; \mathbf{0})$ and Plücker coordinates: ( $L M N ; 000)$. If $\boldsymbol{S}=\mathbf{0}$, the line lies in a plane at infinity, the Plücker vectors: $\left(\mathbf{0} ; \boldsymbol{S}_{\mathbf{0}}\right)$ and Plücker coordinates: $(000 ; P Q R)$. If $\boldsymbol{S} \cdot \boldsymbol{S}^{\mathbf{0}} \neq 0$, that is the vector pair does not satisfy the orthogonality condition, the vector pair forms a screw: $\$=$ $\left(\boldsymbol{S} ; \boldsymbol{S}^{\mathbf{0}}\right), \boldsymbol{S} \cdot \boldsymbol{S}^{\mathbf{0}} \neq 0$. $\mathbf{S}$ vector of screw does not depend from origin, however, $\boldsymbol{S}^{\mathbf{0}}$ depends on the distance from origin. If starting point is moved from origin to point B, then the torque of $\boldsymbol{S}$ around B: $\boldsymbol{S}^{\boldsymbol{B}}=\boldsymbol{S}^{\mathbf{0}}+$ $\boldsymbol{B O} \times \boldsymbol{S}$. Multiply both sides by the vector $\boldsymbol{S}: \boldsymbol{S} \cdot \boldsymbol{S}^{\boldsymbol{B}}=\boldsymbol{S} \cdot \boldsymbol{S}^{\mathbf{0}}+\boldsymbol{S} \cdot(\boldsymbol{B O} \times \boldsymbol{S}) . \boldsymbol{S} \cdot \boldsymbol{S}^{\boldsymbol{B}}=\boldsymbol{S} \cdot \boldsymbol{S}^{\mathbf{0}}$ shows that $\boldsymbol{S} \cdot \boldsymbol{S}^{\mathbf{0}}$ does not depend on the origin. If $\boldsymbol{S} \neq \mathbf{0}$, an origin independent parameter is obtained: $h=$ $\frac{\boldsymbol{S} \cdot \boldsymbol{S}^{\mathbf{0}}}{\boldsymbol{S} \cdot \boldsymbol{S}}$ which is called pitch. $\boldsymbol{S}^{\mathbf{0}}$ is made up of two components, one of them, $h \boldsymbol{S}$ parallel to $\boldsymbol{S}$ axis, the other, 
$\boldsymbol{S}^{\mathbf{0}}-h \boldsymbol{S}$ perpendicular to the $\boldsymbol{S}$ axis. So, the screw: $\$=\left(\boldsymbol{S} ; \boldsymbol{S}^{\mathbf{0}}\right)=(\boldsymbol{S} ; \boldsymbol{r} \times \boldsymbol{S}+h \boldsymbol{S})$. The relation between $\boldsymbol{S}_{\mathbf{0}}$ and $\boldsymbol{S}^{\mathbf{0}}: \boldsymbol{S}^{\mathbf{0}}=\boldsymbol{r} \times \boldsymbol{S}+h \boldsymbol{S}=\boldsymbol{S}_{\mathbf{0}}+h \boldsymbol{S}$. Four factors determine the screw: its position, direction, magnitude, and pitch (Huang et al., 2012).

Screw: $\left(\boldsymbol{S} ; \boldsymbol{S}^{\mathbf{0}}\right): \boldsymbol{S} \neq \mathbf{0}, \boldsymbol{S} \cdot \boldsymbol{S}^{\mathbf{0}} \neq 0,0 \neq h \neq \infty$.

Line vector: $\left(\boldsymbol{S} ; \boldsymbol{S}_{\mathbf{0}}\right): \boldsymbol{S} \neq \mathbf{0}, \boldsymbol{S} \cdot \boldsymbol{S}_{\mathbf{0}}=0, h=0$.

Couple: $(0 ; \boldsymbol{S}): \boldsymbol{S} \neq \mathbf{0}, h=\infty$.

\section{Presentation of the analysed structure}

The tilting table consists of a platform and three actuators of a same construction (A1, A2, A3) and a universal joint (C4) (Figure 2), (Table 1.). The connection of the table to the base is realized by the central universal joint and the actuators (Huang et al., 2013a). Structure of the three actuators: universal joint, prismatic joint, universal joint.

Table 1. Structure of the tilting table

\begin{tabular}{|c|c|c|c|c|}
\hline Markings & 1. Actuator & 2. Actuator & 3. Actuator & $\begin{array}{c}\text { Central universal } \\
\text { joint }\end{array}$ \\
\hline Actuators & $\mathrm{A} 1$ & $\mathrm{~A} 2$ & $\mathrm{~A} 3$ & - \\
\hline Central connection & - & - & - & $\mathrm{C} 4$ \\
\hline Upper universal joint & $\mathrm{A} 1 \mathrm{U}_{\mathrm{U}}$ & $\mathrm{A} 2 \mathrm{U}_{\mathrm{U}}$ & $\mathrm{A} 3 \mathrm{U}_{\mathrm{U}}$ & - \\
\hline Prismatic joint & $\mathrm{A} 1 \mathrm{P}$ & $\mathrm{A} 2 \mathrm{P}$ & $\mathrm{A} 3 \mathrm{P}$ & - \\
\hline Lower universal joint & $\mathrm{A} 1 \mathrm{U}_{\mathrm{L}}$ & $\mathrm{A} 2 \mathrm{U}_{\mathrm{L}}$ & $\mathrm{A} 3 \mathrm{U}_{\mathrm{L}}$ & - \\
\hline
\end{tabular}

The universal joint has two degrees of freedom, it can turn around two axes. The prismatic joint has a degree of freedom and is able to move in a straight line along its axis. The system of the two elements determines the degree of freedom of the structure.

Figure 2 shows a variant of the structure. Due to the structure of the mechanism, the actuators can be placed several arrangements between the platform and the base.

\section{Examination of the installation location of the actuators}

Due to the structural characteristics of the analysed mechanism, the actuators can be built into the structure in several arrangements. The current layout is shown in Figure 3. It can be seen that the central universal joint cuts the platform into four parts, but on closer inspection, it can be noticed that the axis lines do not belong to any of the four parts, so including the axis lines, a total of eight segments can be distinguished. The mechanism is operated by three actuators. The task: how many ways can three actuators be installed in eight segments. There are three cases:

1. All three actuators are located in the same segment.

2. All three actuators are located in separate segments (Figure 3).

3. Two of the three actuators are located in the same segment, the third in another. 


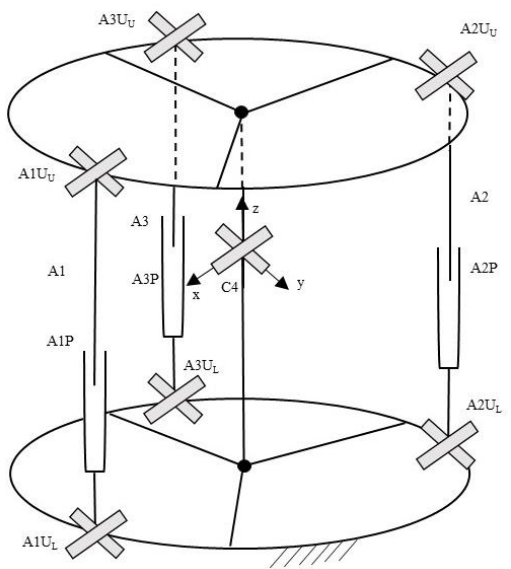

Figure 2. Structure of the tilting table.

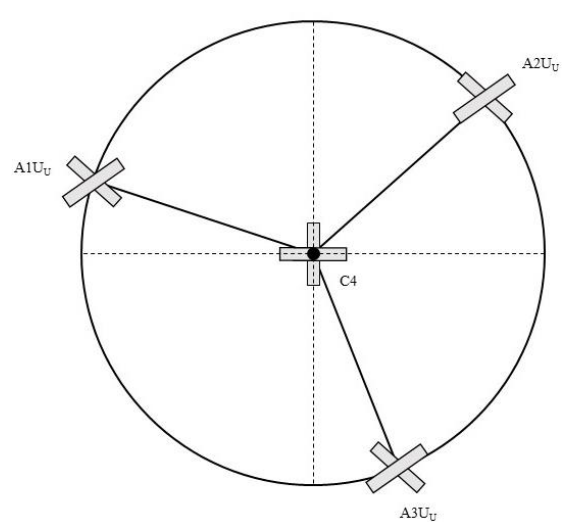

Figure 3. Current arrangement of the tilting table actuators.

The mentioned three cases explore possible installation locations (Mladenović, 2019). The first case is the simplest because the three actuators can only be in the same segment. Since there are eight segments, the number of possible layouts is eight.

In the second case, all three actuators are located in different segments. It cannot be as simple here as in the first case. The task is how many ways can place three objects, actuators in eight segments (boxes). In a more professional form: how many ways can $\mathrm{k}$ elements be selected from $\mathrm{n}$ different elements, if the order of selection does not matter; then a combination of $\mathrm{n}$ elements $\mathrm{k}$-th class without repetition is obtained. $C_{n}^{k}=\frac{n !}{k !(n-k) !}=\frac{8 !}{3 !(8-3) !}=7 \cdot 8=56$. As a result, actuators can be built into the structure in 56 ways.

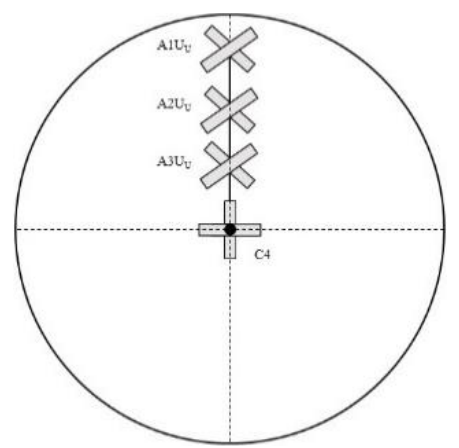

Figure 4. Arrangement of tilting table actuators in the first case.

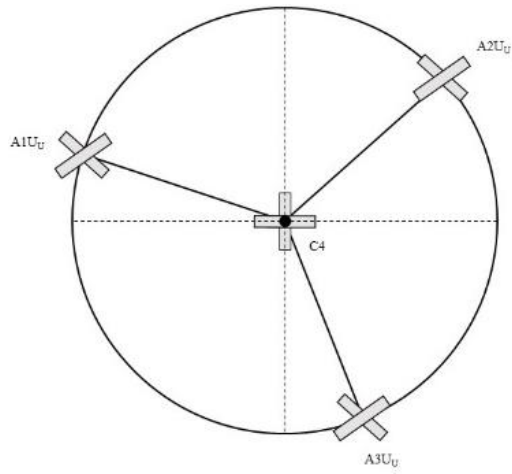

Figure 5. Arrangement of tilting table actuators in the second case.

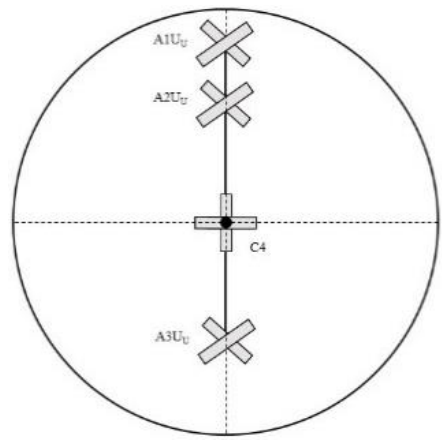

Figure 6. Arrangement of tilting table actuators in the third case.

The third case is a little easier after the second case. The task is how many ways to place two objects into eight segments. In a more professional form: how many ways can $\mathrm{k}$ elements be selected from $\mathrm{n}$ different elements, if the order of selection matters; then a variation of $\mathrm{n}$ elements $\mathrm{k}$-th class without 
repetition is obtained. $V_{n . k}=\frac{n !}{(n-k) !}=\frac{8 !}{(8-2) !}=7 \cdot 8=56$. As a result, in this case, the actuators can be built into the structure in 56 ways. Considering all three cases, it is possible to build actuators into the mechanism in a total of 120 ways. Now, the degree of freedom of all 120 possible mechanism variants will not be calculated. In terms of mobility, one variant is examined from each of the three cases (Huang et al., 2013b).

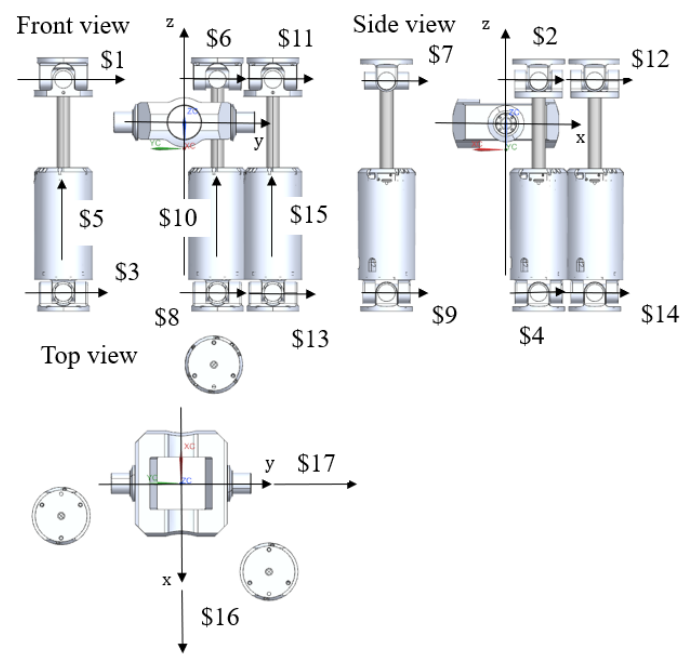

Figure 7. The screw system of the tilting table.

\subsection{The degree of freedom of the mechanism in the first case}

Figure 4 shows that the three actuators fall in a straight line. Production of screw system for actuators and central joint:

Table 2. Screw system for actuators and central connection.

\begin{tabular}{|c|c|c|c|}
\hline $\begin{array}{c}\text { First actuator screw } \\
\text { system }\end{array}$ & $\begin{array}{c}\text { Second actuator screw } \\
\text { system }\end{array}$ & $\begin{array}{c}\text { Third actuator screw } \\
\text { system }\end{array}$ & $\begin{array}{c}\text { Central connection } \\
\text { screw system }\end{array}$ \\
\hline$\$ 1=\left(\begin{array}{llll}0 & 1 & 0 & 0\end{array}-z_{1} 0-x_{1}\right)$ & $\$ 6=\left(\begin{array}{llll}0 & 1 & 0 & 0\end{array}-z_{6} 0-x_{6}\right)$ & $\$ 11=\left(\begin{array}{lll}0 & 1 & 0 ;\end{array}-z_{11} 0-x_{11}\right)$ & \\
\hline 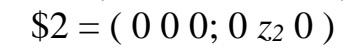 & 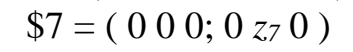 & 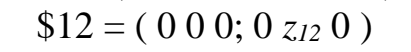 & \\
\hline 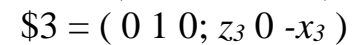 & 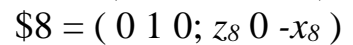 & 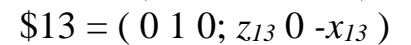 & $00)$ \\
\hline 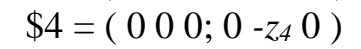 & 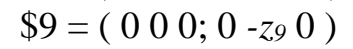 & 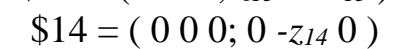 & \\
\hline 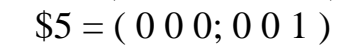 & 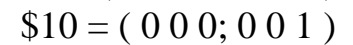 & 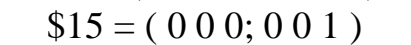 & \\
\hline
\end{tabular}

Production of constraint screw system for the actuators and central connection using the reciprocal condition:

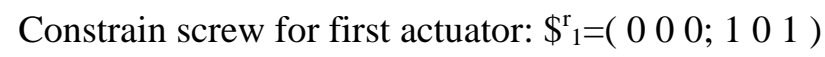

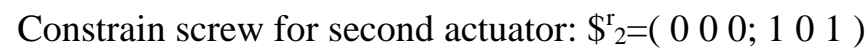

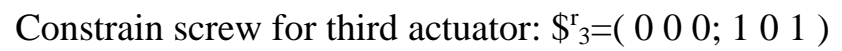

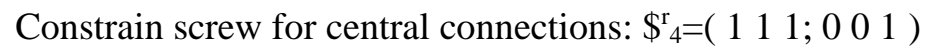


Summary of the constraint screw system of the actuators and central connection:

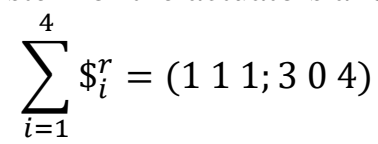

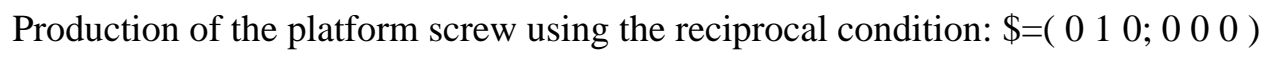

The result shows that the workpiece tilting table has a degree of freedom, it can rotate about the $y$ axes.

\subsection{The degree of freedom of the mechanism in the second case}

Figure 5 shows that the three actuators fall into different segments. Figure 7 shows the screw system marking. Production of screw system for actuators and central joint:

Table 3. Screw system for actuators and central connection.

\begin{tabular}{|c|c|c|c|}
\hline $\begin{array}{c}\text { First actuator screw } \\
\text { system }\end{array}$ & $\begin{array}{c}\text { Second actuator screw } \\
\text { system }\end{array}$ & $\begin{array}{c}\text { Third actuator screw } \\
\text { system }\end{array}$ & $\begin{array}{l}\text { Central connection } \\
\text { screw system }\end{array}$ \\
\hline 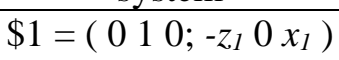 & $\$ 6=\left(\begin{array}{llllll}0 & 1 & 0 & -z_{6} & 0 & x_{6}\end{array}\right)$ & 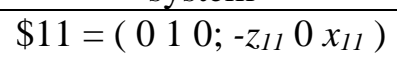 & \\
\hline 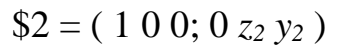 & 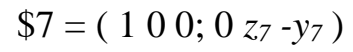 & 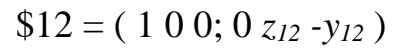 & \\
\hline 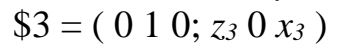 & $\$ 8=\left(\begin{array}{lll}0 & 1 & 0\end{array} z_{8} 0-x_{8}\right)$ & $\$ 13=\left(\begin{array}{llll}0 & 1 & 0 & ; \\
z_{13} & 0 & x_{13}\end{array}\right)$ & 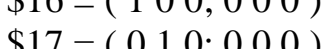 \\
\hline 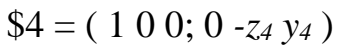 & 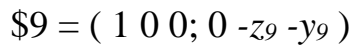 & 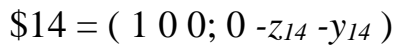 & \\
\hline 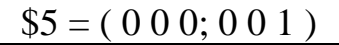 & 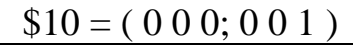 & $\$ 15=\left(\begin{array}{llll}0 & 0 & 0\end{array} ; \begin{array}{lll}0 & 0 & 1\end{array}\right)$ & \\
\hline
\end{tabular}

Production of constraint screw system for the actuators and central connection using the reciprocal condition:

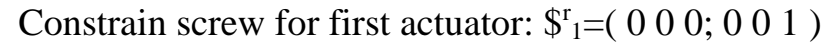

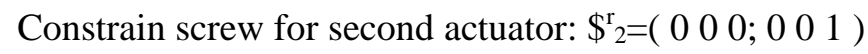

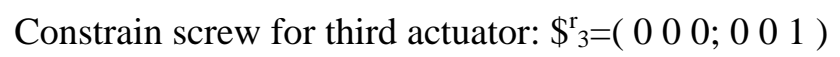

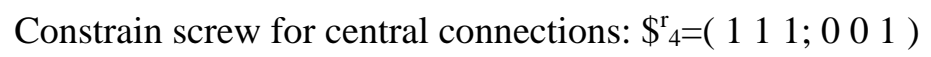

Summary of the constraint screw system of the actuators and central connection:

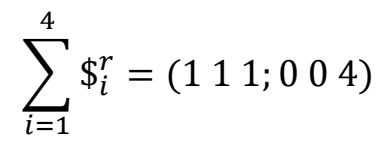

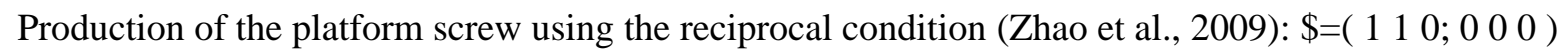

The result shows that the workpiece tilting table has two degrees of freedom, it can rotate about the $\mathrm{x}$ and $\mathrm{y}$ axes.

\subsection{The degree of freedom of the mechanism in the third case}

Figure 6 shows that the three actuators fall into two different segments. Production of screw system for actuators and central joint: 
Table 4. Screw system for actuators and central connection.

\begin{tabular}{|c|c|c|c|}
\hline $\begin{array}{l}\text { First actuator screw } \\
\text { system }\end{array}$ & $\begin{array}{l}\text { Second actuator screw } \\
\text { system }\end{array}$ & $\begin{array}{c}\text { Third actuator screw } \\
\text { system }\end{array}$ & $\begin{array}{l}\text { Central connection } \\
\text { screw system }\end{array}$ \\
\hline$\$ 1=\left(\begin{array}{llll}0 & 1 & 0 & 0\end{array}-z_{1} 0-x_{1}\right)$ & 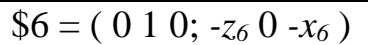 & 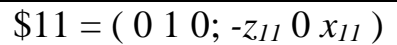 & \\
\hline 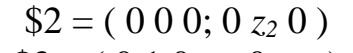 & 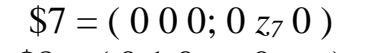 & 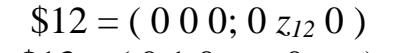 & \\
\hline$\$ 3=\left(\begin{array}{llll}0 & 1 & 0 & ; \\
z_{3} & 0 & -x_{3}\end{array}\right)$ & $\$ 8=\left(\begin{array}{llll}0 & 1 & 0 & ; \\
z_{8} & 0 & -x_{8}\end{array}\right)$ & 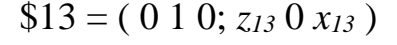 & $\$ 17=(010.000)$ \\
\hline 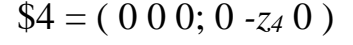 & 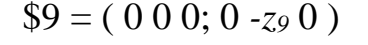 & 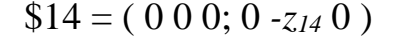 & \\
\hline 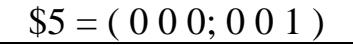 & 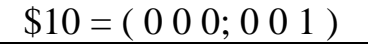 & $\$ 15=\left(\begin{array}{lllll}0 & 0 & 0\end{array} ; \begin{array}{llll}0 & 0 & 1\end{array}\right)$ & \\
\hline
\end{tabular}

Production of constraint screw system for the actuators and central connection using the reciprocal condition:

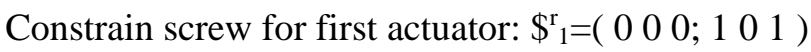

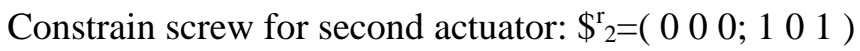

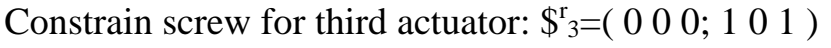

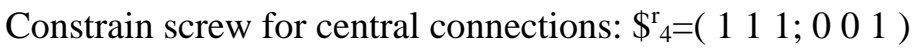

Summary of the constraint screw system of the actuators and central connection:

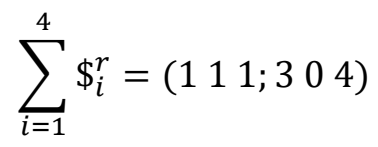

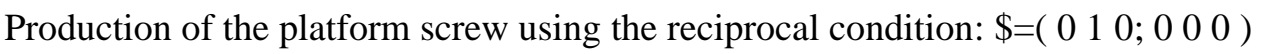

The result shows that the workpiece tilting table has a degree of freedom, it can rotate about the y axes.

\section{Summary}

The paper presents an investigation of a parallel kinematic chain mechanism. The analysis is complex and consists of two parts. First, the combinatorics are used to determine the combinations and variations of the possible installation locations of the actuators. Afterward the degree of freedom of the structure is determined for three selected versions of possible combinations and variations. The results well reflect that the installation location of the actuators plays an important role in the functionality of the mechanism. In the first and third cases the structure has only one degree of freedom, therefore it is not suitable for operating the platform. While the second case provides installation locations where the mechanism has two degrees of freedom, it is suitable for operating the platform.

\section{References}

[1] Bas, E. (2019). Basics of probability and stochastic processes. Vol. 1. Springer. https://doi.org/10.1007/978-3-030-32323-3

[2] Marshall, G. S. (1998). Vector algebra. Introductory mathematics: Applications and methods. 2132. https://doi.org/10.1007/978-1-4471-3412-1_2

[3] Szeidl, Gy. (2014). A vektor- és tenzorszámítás alapismeretei mérnököknek. Müszaki mechanika.

[4] Huang, Z., Li, Q., \& Ding, H. (2012). Basics of screw theory. Mechanisms and Machine Science. 1-16. Springer. https://doi.org/10.1007/978-94-007-4201-7_1 
[5] Huang Z., Li Q., Ding H. (2013a). Mobility analysis Part-1., Theory of parallel mechanisms. Mechanisms and Machine Science. Vol. 3. Springer. https://doi.org/10.1007/978-94-007-4201-7_3

[6] Mladenović, P. (2019). Combinatorics. Problem Books in Mathematics. Vol. 2. Springer. https://doi.org/10.1007/978-3-030-00831-4

[7] Huang Z., Li Q., Ding H. (2013b). Constraint screw-based method for type synthesis. Theory of Parallel Mechanisms, Mechanisms and Machine Science. Vol 9. Springer. https://doi.org/10.1007/978-94-007-4201-7_9

[8] Zhao, J., Li, B., Yang, X. and Yu, H. (2009). Geometrical method to determine the reciprocal screws and applications to parallel manipulators. Robotica, 27(6), 929-940. https://doi.org/10.1017/S0263574709005359 